\title{
A MODEL IN CONTINUOUS TIME AND SPACE TO STUDY ECONOMIC MIGRATION
}

\author{
Carmen Camacho ${ }^{1, *}$ and Agustín Pérez-Barahona ${ }^{2,3}$
}

\begin{abstract}
Population movements modify the environment, land-use, and shape the landscape through urbanization. Furthermore, migration has become one of the most relevant determinants of global human health and social development. The objective of this paper is to provide a framework to understand the economic and natural factors responsible for migration and population agglomerations and their environmental consequences. In this regard, we develop an economic model in continuous time and space adapting Hotelling's migration law to make individuals react to possible improvements of their welfare. First we show that there is solution to this spatial-dynamic problem. Then, we illustrate the properties of the economy and the associated population dynamics through numerical simulations.
\end{abstract}

Mathematics Subject Classification. 49J20, 49K20, 91B55, 91B62, $91 \mathrm{~B} 72$.

Received March 2, 2018. Accepted December 13, 2018.

\section{INTRODUCTION}

Changes in the spatial distribution of humans trigger modifications in geography and shape the environment. Moreover, migration has become one of the most relevant determinants of global human health and the associated social development. Among the many factors that explain human migration the economic literature points out unemployment and wage differentials [23, 26], political factors such as wars and prosecution [37], and environmental problems such as floods, draughts or the sea level rise [1, 20, 24, 28]. In this paper we provide a model à la Solow in which the economic reasons that trigger human migration are well founded and evolve with the human flow and the environmental quality. In contrast to previous papers such as Volpert et al. [39], the environmental conditions are endogenously determined, encompassing spatial population dynamics and economic activity. Our setting is continuous in space and time. Therefore, short and long-run motives, population configurations and their consequences can be analysed from a local, regional and global perspective.

Migration flows are paramount in contemporary economies. In order to understand the role played by this source of population dynamics, a number of authors have proposed models with labor movements. Many consider partial equilibrium analysis, where the dynamic implications of migrations on the regional economy at origin and destination are absent. As a consequence, they cannot analyse the short and medium effects of population movement (see among others $[6,7,10,11,30]$ ). Only few models encompass migrations and their effects on the

Keywords and phrases: Migration, spatial dynamics, economic growth, parabolic PDE.

${ }^{1}$ CNRS at Paris School of Economics, 48 Boulevard Jourdan, 75014 Paris, France.

2 THEMA, University of Cergy-Pontoise, 95011 Cergy-Pontoise, France.

3 Ecole Polytechnique, 91128 Palaiseau, France.

* Corresponding author: carmen.camacho@psemail.eu 
economy both in the short and the long term. Most of them belong to the New Economic Geography literature, which aims at understanding uneven development and the emergence of economic agglomerations. Fujita and Thisse [17] develop a two region - three sector model in which skilled workers can migrate. They find that the symmetric distribution of workers is the unique equilibrium, but it is unstable. In Krugman [22] and Fukao and Benabou [15] agents compare current salaries to decide whether to migrate or not. They also conclude that the symmetric equilibrium is unstable. Gianetti [19] proposes a general equilibrium framework where individuals' skills are complementary. This induces self-selection in migration. If the skill premium increases with average human capital, then skilled workers will move to rich regions. In the long-run labor is segregated by skill.

Some research has also utilized continuous space settings following the pioneering works of Hotelling [21], von Thünen [38] and Salop [33]. Fujita et al. [15] describe the evolution of sectoral employment in a circle. Following Turing [36], they assume an initial uniform distribution of labor among sectors and at all locations. If the economy receives a sufficiently large shock, then two agglomerations can surge in opposite locations. Mossay [27] studies the role of expectations formation in economic settings with continuous space. If initial endowments are sufficiently unequal, rational adjustments can promote convergence.

Our paper reconsiders Hotelling (1929)'s [21] approach. Hotelling proposed a provocative vision of economics on space, underlining the diffusion nature for human migration. Under this lens, individuals react to changes in salaries and move like a flow. In 1951, Skellam rediscovered and applied the very same migration law in biology. Skellam [34] proposes distinct diffusion laws for the spatial dynamics of forests and various species of small animals. In this set-up population diffusion depends on the population concentration together with the fertility and mortality rates, which are location specific. Noteworthy, the resulting dynamics coincide with the trajectories of a model in which individuals move randomly, that is, following a Brownian motion. Based on Hotelling's law, Beckmann and Puu [4] and Puu [31] modeled population diffusion as a function of spatial productivity differentials. Puu [32] integrates Skellam's law into his model of Puu [31]. This time there are two forces in place. First, population moves towards less populated areas. And second, population moves towards areas offering higher wages.

Also building on Hotelling [21], some recent authors have further analyzed the economic motives that urge people to migrate. Beckmann [5] enunciates and solves the spatial problem presented by Hotelling assuming a convex-concave production function. Two works are particularly close to ours. Alvarez and Mossay [3] propose a migration law which captures three decisive demographic factors: population growth, population diffusion and migration drift towards preferred regions. They additionally consider climate and economic outcomes as relevant factors in migration. However in this setting population movements do not affect the economic situation. In other words, the interaction between population spatial dynamics and economic outcomes is neglected.

From a mercantilistic perspective, Volpert et al. [39] introduce a model where population is attracted by the wealth accumulated in each location. Locations create new wealth by combining natural resources, labour and physical capital. Natural resources are exogenous, while the levels of labour and physical capital are assumed to be proportional to location's population density and wealth, respectively. Consumption, which is also considered as proportional to population density and wealth, directly reduces the stock of wealth of the location. This framework allows for the diffusion of population and wealth across locations. However, in contrast to the previous literature, it additionally includes cross-diffusion effects of both state variables: wealth differences between locations induce migration, implying in turn the transportation of part of the wealth accumulated. With this model in hand, they put forward spatio-temporal patterns of population in the form of travelling waves.

All the aforementioned literature assumes that individuals follow demographic flows, or at best a combination of environmental and economic conditions. In the present work we conduct a similar exercise, although we endogenize locations' economy. Indeed, since production requires labor, the final outcome at each location depends on the size of its population and hence on migration. In order to emphasize the role of environmental conditions in contemporary economies, we also consider that environmental quality is an additional production input. This feature particularly allows us to illustrate the possible environmental degradation due to population movements. We prove the existence of solution to this spatial-dynamic framework. 
Discretizing the corresponding system of partial differential equations (PDE), we use an algorithm to numerically illustrate the dynamic characteristics of the economy. We study how initial spatial inequalities, i.e., first nature causes [9, 16], evolve in time, inducing different geographical patterns. We explore the importance of initial endowments, both in terms of labor and the environmental quality. Although migration smoothes out spatial differences, we find that if the environmental quality is not evenly distributed it can trigger everlasting inequalities. In this respect, our results show that diffusion does not play a major qualitative role. Instead, its role is mainly quantitative, accelerating or decelerating the dynamics of population. Regarding the role played by heterogeneity in total factor productivity and in environmental sensitivity, we identify the existence of opposite migration forces. We distinguish between primary forces that privilege the agglomeration of individuals to increase production, and secondary forces that affect individuals utility in various ways, such as technological disparities. Finally, we complete our numerical analysis by including the environmental quality into individuals' welfare. Richer dynamics arise, including travelling waves.

The article is structured as follows. We present our version of Hotelling's migration law in Section 2. Section 3 sets the spatial-dynamic framework, which specifies the migration law considered for this paper. Although in Section 3 the geographical space is one-dimensional, Section 4 proves that there is solution to a generalized problem in which space is M-dimensional with $M \geq 1$. Then, we numerically illustrate the spatial dynamics of our economy in Section 5. Finally, Section 6 concludes.

\section{A Version of Hotelling's Migration LAW}

Hotelling [21] migration law postulates that individuals move according to the gradient of salaries. In this logic, individuals move towards the locations offering the highest salaries. As mentioned in the introduction, this modelling strategy was also adopted in Puu [31], Beckman and Puu [4] and Beckman [5]. Denoting by $h(x, t)$ the population at location $x$ and time $t$, the dynamics of population are described according to Hotelling by the following parabolic PDE:

$$
h_{t}(x, t)-h_{x x}(x, t)=w_{x}(x, t),
$$

where $w(x, t)$ denotes the salary per unit of labor at location $x$ and time $t, w_{x}$ is the spatial derivative of $w$ and, as usual, we adopt the notation $h_{t} \equiv \frac{\partial h(x, t)}{\partial t}, h_{x x} \equiv \frac{\partial^{2} h(x, t)}{\partial x^{2}}$. Here $t \in[0, T]$ and $x \in \Sigma \subseteq \mathbb{R}$.

In contrast to Hotelling's migration law, we assume that individuals move following the gradient of their utility as in Ottaviano [29]. Individuals move towards locations that provide higher utility or higher instantaneous felicity, that we call $u$. We specify $u$ in Section 3. Under this assumption, the resulting spatial-dynamic law of human capital is

$$
h_{t}(x, t)-d h_{x x}(x, t)=u_{x}(x, t),
$$

where $d \in \mathbb{R}$ is the diffusion parameter. Notice that introducing a parameter to account for diffusion allows us to consider different degrees of population mobility. For $d \geq 0$, a low value of the diffusion parameter can be identified with the existence of barriers to migration. It could also be interpreted as the percentage of people willing to migrate and who succeeds.

\section{A spatial MODEL TO ACCOUNT FOR MigRATION}

We set a model to describe individuals migration in a bounded region of space as a response to changes in their utility. The initial distribution of population over $\Theta \subset \mathbb{R}$ is known. Production of the unique consumption good, $Y$, requires labour and environmental quality. As observed in the introduction, the latter input incorporates the effect of the environmental degradation induced by population mobility. In our simple set-up we use a wide definition of environmental quality, which can also include the availability of natural resources as in Mariani et al. [25]. Following the standard economic literature, inputs transform into consumption good through a 
production function $\mathcal{F}$ :

$$
Y(x, t)=A(x, t) \mathcal{F}(h(x, t), r(x, t)),
$$

where $A$ is total factor productivity and $r$ represents the environmental quality of location $x$ at time $t$. $\mathcal{F}$ satisfies the following assumption:

Assumption 3.1. $\mathcal{F}$ is a positive, increasing, differentiable function of population and the environmental quality:

$$
\mathcal{F}(h, r) \geq 0, \text { and } \mathcal{F}_{h}^{\prime}(h, r) \geq 0, \mathcal{F}_{r}^{\prime}(h, r) \geq 0,
$$

for all $(h, r)$. Furthermore, $\mathcal{F}(h, r)>0$ for $h, r>0$.

Assumption 3.1 implies that the production function is strictly positive for interior solutions, and that the larger the labour force and/or the environmental quality the larger the production.

Without loss of generality, the price of the consumption good is normalized to 1 . Assuming that there is no trade in the final good, individuals can only consume the production of their own location.

The environmental quality is damaged by the location's production:

$$
r_{t}(x, t)=-\delta Y(x, t)+\gamma r(x, t),
$$

where $\delta$ represents the environmental degradation induced by each unit of consumption and $\gamma \in[0,1]$ is the natural regeneration rate of the environment. The initial environmental quality is known. Note that in contrast to the population, the environmental quality does not move in space, that is $r_{x}(x, t)=0$. We consider this simplification in order to concentrate on the movement of population. ${ }^{1}$

As frequently assumed in the economic literature, individuals measure their welfare by means of a utility function $u$, which depends solely on consumption and it verifies ${ }^{2}$

Assumption 3.2. $u$ is a positive, increasing and concave function of individual's consumption, $c$. $u$ is twice differentiable and

$$
u(c) \geq 0, \quad u^{\prime}(c) \geq 0 \text { and } u^{\prime \prime}(c) \leq 0, \text { for all } c \geq 0
$$

With these elements in hand, we can rewrite the migration law in (2.2) as

$$
h_{t}(x, t)-d h_{x x}(x, t)=u^{\prime}(c(x, t)) c_{x}(x, t) .
$$

We can substitute $c(x, t)=\frac{A(x, t) \mathcal{F}(h(x, t), r(x, t))}{h(x, t)}$ in (3.2) to obtain:

$$
h_{t}-d h_{x x}=u^{\prime}\left(A \frac{\mathcal{F}(h, r)}{h}\right)\left[A_{x} \frac{\mathcal{F}(h, r)}{h}+A \frac{\mathcal{F}_{h}^{\prime}(h, r)}{h} h_{x}+A \frac{\mathcal{F}_{r}^{\prime}(h, r)}{h} r_{x}-A \frac{\mathcal{F}(h, r)}{h} \frac{h_{x}}{h}\right] .
$$

where $A_{x}=\frac{\partial A}{\partial x}$ and $h_{x}=\frac{\partial h}{\partial x}$. Gathering the partial derivatives of $h$ on the left hand side, we can rewrite (3.3) as

$$
h_{t}-d h_{x x}-u^{\prime}\left(A \frac{\mathcal{F}(h, r)}{h}\right)\left(A \frac{\mathcal{F}_{h}^{\prime}(h, r)}{h} h_{x}+A \frac{\mathcal{F}_{r}^{\prime}(h, r)}{h} r_{x}-A \frac{\mathcal{F}(h, r)}{h} \frac{h_{x}}{h}\right)=u^{\prime}\left(A \frac{\mathcal{F}(h, r)}{h}\right) A_{x} \frac{\mathcal{F}(h, r)}{h} .
$$

\footnotetext{
${ }^{1}$ Camacho and Pérez-Barahona [12] provide a spatial-dynamic set-up where natural resources move across locations. Nevertheless, this hypothesis applies to many natural resources such as forest, agricultural land, lakes, aquifers, etc.

${ }^{2}$ In Section 5.5 we study numerically the model with a utility function that depends on both consumption and environmental quality.
} 
In addition to (3.4), the initial distribution of population $h_{0}(x) \equiv h(x, 0)$ and the initial environmental quality $r_{0}(x) \equiv r(x, 0)$, we need boundary conditions to describe the behavior of the state variable at $\delta \Theta$, the border of $\Theta$. Hence, our objective is to ensure the existence of the solution to

$$
O\left\{\begin{array}{l}
h_{t}-d h_{x x}-u^{\prime}\left(A \frac{\mathcal{F}(h, r)}{h}\right)\left(A \frac{\mathcal{F}_{h}^{\prime}(h, r)}{h} h_{x}+A \frac{\mathcal{F}_{r}^{\prime}(h, r)}{h} r_{x}-A \frac{\mathcal{F}(h, r)}{h} \frac{h_{x}}{h}\right)=u^{\prime}\left(A \frac{\mathcal{F}(h, r)}{h}\right) A_{x} \frac{\mathcal{F}(h, r)}{h}, \\
r_{t}(x, t)=-\delta A \mathcal{F}(h, r)+\gamma r(x, t), \\
h_{\delta \Sigma}=a(t) \\
r_{\delta \Sigma}=b(t),
\end{array}\right.
$$

being $a(t)$ and $b(t)$ known functions $\forall(x, t) \in \Sigma \times(0, T]$. Note first that there is no second order spatial crosseffect in $O$, i.e. there is no $h_{x t}$ nor $r_{x t}$. It is a key feature of our modelling and we will convert this observation into an assumption in next section. Second, the PDE governing population dynamics involves a non-linear operator associated to the first order derivatives. This non-linearity needs to be treated carefully. Most of the results proving the existence of a solution to systems of parabolic PDE are specific to linear operators. In the following section, we will adapt the existence result in Crandall et al. [14] to problems like $O$ defined over a M-dimensional space.

\section{EXISTENCE OF A SOLUTION}

Next, we extend in a simple manner Crandall et al. [14] to prove the existence of a solution to a Dirichlet problem describing the evolution of a $N$ dimensional variable, $u$, defined over $\Omega \subseteq \mathbb{R}^{M}$. The dynamics of the variable is defined by $F$. We assume that $N \geq 1, F: \Omega \times \mathbb{R}^{N} \times \mathcal{M}_{N \times M} \times \mathcal{S}(M)^{N} \rightarrow \mathbb{R}^{N}$, where $D u \in$ $\mathcal{M}_{N \times M}$ is a matrix of size $N \times M$, which collects the partial derivatives of $u=\left(u_{1}, u_{2}, \ldots, u_{N}\right)$ with respect to $x=\left(x_{1}, \ldots, x_{M}\right)$. With an abuse of notation, we define $D^{2} u=\left(D^{2} u_{1}, \ldots, D^{2} u_{N}\right)$, where $D^{2} u_{i}$ is the Hessian matrix of $u_{i}$. Hence, $D^{2} u$ is a collection of $\mathrm{N}$ diagonal square matrices of dimension $M$, i.e. $D^{2} u_{i} \in \mathcal{S}(M)$, for $i=1, \ldots, N$. Hence our problem is

$$
\mathcal{D}\left\{\begin{array}{l}
F\left(x, u, D u, D^{2} u\right)=0, \text { in } \Omega \subseteq \mathbb{R}^{N}, \\
u=0, \text { on } \delta \Omega .
\end{array}\right.
$$

A solution $u$ to this problem is a function $u \in C(\bar{\Omega}), u: \Omega \rightarrow \mathbb{R}^{N}$, that is a viscosity function of $F=0$ in $\Omega$ and satisfies $u(x)=0$, for $x \in \delta \Omega$. We will need the following assumptions:

Assumption 4.1. We assume $\mathbb{R}^{N}$ is endowed with the usual Euclidean norm so that $F$ is defined over a topological space.

Assumption 4.2. $F: \Omega \times \mathbb{R}^{N} \times \mathcal{M}_{N \times M} \times \mathcal{S}(M)^{N} \rightarrow \mathbb{R}^{N}$, is a continuous and proper function, with non-empty compact values.

Note that in order to ensure the existence of a solution we do not need the usual neoclassical hypothesis on $F$. Indeed, we only require that $F$ is proper. We say that a function $F$ is proper if

$$
F(x, r, p, X) \leq F(x, s, p, Y),
$$

whenever $r \leq s$ with $r, s \in \mathbb{R}^{N}$ and $Y \leq X$, that is $Y_{i} \leq X_{i}$ for $i=1, \ldots, N, X_{i}, Y_{i} \in \mathcal{S}(M)$. Regarding order in $\mathcal{S}(M)$, we assume that $\mathcal{S}(M)$ is equipped with the Löwer ordering or positive semi-definite ordering.

Definition 4.3. For any two matrices $A, B \in \mathcal{S}(M), A$ is larger than $B$ in the sense of Löwer and we write $A \geq B$, if and onnly if $v^{T} A v \geq v^{T} B v$, for all $v \in \mathbb{R}^{N}$. 
We also need some regularity of the solution $u$ we are looking for:

Assumption 4.4. $u: \Omega \rightarrow \mathbb{R}^{N}$ is continuous, with non-empty compact values.

In order to prove the existence of a solution, we follow Perron's method. First, let us define the upper hemicontinuous envelope of a function $w(x)$ as

$$
w^{*}(x)=\lim \sup _{r \rightarrow 0}\{w(y): y \in \Omega \text { and }|x-y|<r\} .
$$

Similarly, the lower hemicontinuous envelope of a function $w(x)$ is defined as

$$
w_{*}(x)=\lim \inf _{r \rightarrow 0}\{w(y): y \in \Omega \text { and }|x-y|<r\} .
$$

Perron's method is based on the following theorem:

Theorem 4.5. Let comparison hold for $\mathcal{D}$ in $\Omega$, that is, if $w$ is a subsolution of $D P$ and $v$ is a supersolution of $\mathcal{D}$, then

$$
w \leq v
$$

that is, $w_{j} \leq v_{j}$ for $j=1, \ldots, N$. Suppose there exists a subsolution $\underline{u}$ and a supersolution $\bar{u}$ of DP that satisfy that

$$
\underline{u}_{*}(x)=\bar{u}^{*}(x)=0, \text { for } x \in \delta \Omega .
$$

Then

$$
W(x)=\sup \left\{w(x): \underline{u}_{j} \leq w_{j} \leq \bar{u}_{j} \text {, and } w \text { is a subsolution of } \mathcal{D}\right\}
$$

is a solution of $\mathcal{D}$.

Before dealing with the proof of the theorem, we need some preliminaries which are presented in Section 4.1. The theorem is proved after, in Section 4.2. Section 4.3 seeks for an upper and a lower solution to $O$ under some assumptions on the functional forms. We assume those forms that will be utilized in the numerical exercises of Section 5.

\subsection{Preliminaries}

In the proof of Theorem 4.5, we invoke Berge's Maximum Theorem to ensure that a function reaches a maximum $^{3}$ :

Theorem 4.6 (Berge's Maximum Theorem). Let $\Psi: X \rightarrow Y$ be a continuous correspondence between topological spaces with non-empty compact values, and suppose $f: G r \Psi \rightarrow \mathbb{R}$ is continuous. Define the value function $m: X \rightarrow \mathbb{R}$ by

$$
m(x)=\max _{y \in \Psi(x)} f(x, y)
$$

and the correspondence $\mu: X \rightarrow Y$ of maximizers by

$$
\mu(x)=\{y \in \Psi(x): f(x, y)=m(x)\} .
$$

\footnotetext{
${ }^{3}$ This version of Berge's Maximum Theorem is extracted from Aliprantis and Border [2].
} 
Then:

(i) The value function $m$ is continuous.

(ii) The argmax correspondence $\mu$ has non-empty compact values.

(iii) If either $f$ has a continuous extension to all of $X \times Y$ or $Y$ is Hausdorff, then the argmax correspondence $\mu$ is upper-hemicontinuous.

If function $\psi$ is also $C^{2}$ with non-empty compact values, then $u-\psi$ satisfies Berge's assumption and $u-\psi$ reaches a local maximum at $\hat{x}$. Hence, $D u=D \psi$ and $D^{2} u_{i} \leq D^{2} \psi_{i}$ in the sense of Löwer, for all $i$. If we abuse again of notation, let us denote $D^{2} \psi=\left(D^{2} \psi_{1}, \ldots, D^{2} \psi_{N}\right)$ the vector made of the Hessian matrices associated to $\psi=\left(\psi_{1}, \ldots, \psi_{N}\right)$.

Note that since the function $F$ defining our problem $\mathcal{D}$ is proper, then

$$
F\left(\hat{x}, u(\hat{x}), D \psi(\hat{x}), D^{2} \psi(\hat{x})\right) \leq F\left(\hat{x}, u(\hat{x}), D u(\hat{x}), D^{2} u(\hat{x})\right) \leq 0 .
$$

Therefore, if $u$ is a subsolution of $F=0$ then

$$
F\left(\hat{x}, u(\hat{x}), D \psi(\hat{x}), D^{2} \psi(\hat{x})\right) \leq 0,
$$

whenever $\psi \in C^{2}$ and $\hat{x}$ is a local maximum. In the proof, we apply numerous times Taylor approximations. Let us recall here a version of Taylor's Theorem:

Theorem 4.7. Let $f: \mathbb{R}^{N} \rightarrow \mathbb{R}^{N}$, at least twice continuously differentiable. We can approximate the value of the function $f$ at $y$ in $\mathbb{R}^{N}$ using the known values $f(x), \nabla f(x)$ and $H[f(x)]$ for $x$ in $\mathbb{R}^{N}$ sufficiently close to $y$ :

$$
f_{i}(y)=f_{i}(x)+\nabla f_{i}(x)^{T}(y-x)+\frac{1}{2}(y-x)^{T} H\left[f_{i}(x)\right](y-x)+o\left(|y-x|^{2}\right),
$$

where $\nabla f_{i}(x)^{T}=\left(\frac{\partial f_{i}}{\partial x_{1}}, \ldots, \frac{\partial f_{i}}{\partial x_{N}}\right)$ and $H\left[f_{i}(x)\right]$ is the Hessian matrix of function $f_{i}$ evaluated at $x$ for all $i=1, \ldots, N$.

Making use of Taylor's Theorem, we can write that for every $i=1, \ldots, N$

$$
u_{i}(x)=u_{i}(\hat{x})+\nabla u_{i}(\hat{x})^{T}(x-\hat{x})+\frac{1}{2}(x-\hat{x})^{T} H\left[u_{i}(x-\hat{x})\right](x-\hat{x})+o\left(|x-\hat{x}|^{2}\right) .
$$

Hence if $x$ is sufficiently close to $\hat{x}$, and since $D u_{i}(\hat{x})=D \psi_{i}(\hat{x})$ then condition $\mathcal{C}$ below holds

$$
\mathcal{C}: u_{i}(x) \leq u_{i}(\hat{x})+\nabla \psi_{i}(\hat{x})^{T}(x-\hat{x})+\frac{1}{2}(x-\hat{x})^{T} H\left[\psi_{i}(x-\hat{x})\right](x-\hat{x}),
$$

for every $i=1, \ldots, N$. Let us introduce the following notation $p=\left(D \psi_{1}, \ldots, D \psi_{N}\right), X=\left(D^{2} \psi_{1}, \ldots, D^{2} \psi_{N}\right)$. Following Crandall et al. [14], we define a subsolution $u$ of $F=0$, as a function that satisfies

$$
F(\hat{x}, u(\hat{x}), p, X) \leq 0
$$

when $\psi$ is $C^{2}$ and $\hat{x}$ is a local maximum of $u-\psi$.

We need to define the set $\Theta \subseteq \Omega \subseteq \mathbb{R}^{M}$, on which we can find $x$ such that $F \leq 0$. We define $J_{\Theta}^{2,+} u(\hat{x})$ in relation to $\hat{x}$ :

Definition 4.8. If $u: \Theta \rightarrow \mathbb{R}^{N}$, then $J_{\Theta}^{2,+} u(\hat{x}): \Theta \rightarrow \mathcal{M}_{N \times M} \times \mathcal{S}(M)^{N}$ is the second-order superjet of $u$ at $\hat{x}$, made of the couples $(p, X)$ that satisfy condition $\mathcal{C}$. 


\subsection{An existence theorem}

This proof proceeds in two steps and several lemmas:

Lemma 4.9. Let $\Theta \subset \mathbb{R}^{M}$ be locally compact, $F$ a lower semicontinous function defined over $\Theta \times \mathbb{R}^{N} \times$ $\mathcal{M}_{N \times M} \times \mathcal{S}(M)^{N}$, and $\mathcal{F}$ be a family of solutions of $F \leq 0$ in $\Theta$. Let

$$
w(x)=\sup \{u(x): u \in \mathcal{F}\},
$$

and assume that the upper semicontinuous envelope of $w, w^{*}$ is finite. Then $w^{*}$ is a solution of $F \leq 0$ in $\Theta$.

That is, the upper semicontinuous envelope of $w, w^{*}$, is a solution. The lemma takes the supremum of a family of functions and then it smoothes it. Lemma 4.9 shows that the resulting function is a subsolution of $F=0$. The proof of Lemma 1 comes later, first we need the following proposition:

Proposition 4.10. Let $\Theta \subset \mathbb{R}^{M}$ be locally compact, $v$ an upper semicontinuous function defined on $\Theta$, $z \in \Theta$ and $(p, X) \in J_{\Theta}^{2,+} v(z)$, with $p \in \mathcal{M}_{N \times M}$ and $X \in S(M)^{N}$. Suppose that $u_{n}$ is a sequence of upper semicontinuous functions on $\Theta$ such that

(i) there exists a sequence $x_{n} \in \Theta$ such that $\left(x_{n}, u_{n}\left(x_{n}\right)\right) \rightarrow(z, v(z))$;

(ii) if $z_{n} \in \Theta$ for all $n$ and $z_{n} \rightarrow x \in \Theta$, then $\limsup _{n \rightarrow \infty} u_{n}\left(z_{n}\right) \leq v(z)$. Then, for every $n$ there exists $\hat{x}_{n} \in \Theta$, $\left(p_{n}, X_{n}\right) \in J_{\Theta}^{2,+} u_{n}\left(\hat{x}_{n}\right)$ with $p_{n} \in \mathbb{R}^{N}$ and $X_{n} \in S(M)^{N}$, such that

$$
\left(\hat{x}_{n}, u_{n}\left(\hat{x}_{n}\right), p_{n}, X_{n}\right) \rightarrow(z, v(z), p, X) .
$$

Proof of Proposition 4.10. Without loss of generality, we can assume that $z=0$. Then, for every $\delta=$ $\left(\delta_{1}, \ldots, \delta_{N}\right)$, with $\delta_{i}>0$, there is a $r>0$ such that

$$
N_{r}=\{x \in \Theta:|x| \leq r\}
$$

is compact, and that for $x \in N_{r}$ and for every $i=1, \ldots, N$

$$
v_{i}(x) \leq v_{i}(0)+\left\langle p_{i}, x\right\rangle+\frac{1}{2}\left\langle X_{i} x, x\right\rangle+\delta_{i}|x|^{2} .
$$

We know that there exists a sequence $\left\{x_{n}\right\}_{n}$, with $x_{n} \in \Theta, \forall n$ such that

$$
\left(x_{n}, u_{n}\left(x_{n}\right)\right) \rightarrow(0, v(0)) .
$$

By Berge's Maximum Theorem, the following function

$$
u_{i, n}(x)-\left(\left\langle p_{i}, x\right\rangle+\frac{1}{2}\left\langle X_{i} x, x\right\rangle+2 \delta_{i}|x|^{2}\right)
$$

has a maximum over $N_{r}, \hat{x}_{n} \in N_{r}$, and this is true for every $i$. Then since $\hat{x}_{n}$ is a maximum

$$
\mathcal{E}: u_{i, n}(x) \leq u_{i, n}\left(\hat{x}_{n}\right)+\left\langle p_{i}, x-\hat{x}_{n}\right\rangle+\frac{1}{2}\left(\left\langle X_{i} x, x\right\rangle-\left\langle X \hat{x}_{n}, \hat{x}_{n}\right\rangle\right)+2 \delta_{i}\left(|x|^{2}-\left|\hat{x}_{n}\right|^{2}\right),
$$

for any other $x \in N_{r}$.

In this proposition, we prove the convergence of

$$
\left(\hat{x}_{n}, u_{n}\left(\hat{x}_{n}\right), p_{n}, X_{n}\right) \rightarrow(z, v(z), p, X)
$$


element by element. Note that first it had been assumed that $z=0$ without loss of generality. Then, it has been proven that $\hat{x}_{n} \rightarrow 0$. In what follows, we prove that $u_{n}\left(\hat{x}_{n}\right) \rightarrow v(0)$. We begin by proving that $\lim \sup u_{i, n}(\hat{x}) \leq$ $v_{i}(0)$, for every $i=1, \ldots, N$.

Suppose that (passing to a subsequence if necessary) there exists a convergent subsequence within $\left\{\hat{x}_{n}\right\}_{n}$. Let us assume that the limit of this sequence is $y .{ }^{4}$ Since condition $\mathcal{E}$ holds for every $x \in N_{r}$, will hold for any $x_{n}$ in the theorem, since $x_{n} \in N_{r}, \forall n$. Recall that $x_{n} \rightarrow 0$. We can replace $x$ by $x_{n}$ in inequality $\mathcal{E}$ above to obtain:

$$
u_{i, n}\left(x_{n}\right) \leq u_{i, n}\left(\hat{x}_{n}\right)+<p_{i}, x_{n}-\hat{x}_{n}>+\frac{1}{2}\left(<X_{i} x_{n}, x_{n}>-<X_{i} \hat{x}_{n}, \hat{x}_{n}>\right)+2 \delta_{i}\left(\left|x_{n}\right|^{2}-\left|\hat{x}_{n}\right|^{2}\right)
$$

Recall that by assumption, $x_{n} \rightarrow 0$ and that $\hat{x}_{n} \rightarrow y$, then we can pass to the limit inferior when $n \rightarrow \infty$ :

$$
v_{i}\left(x_{n}\right) \leq \lim \inf _{n \rightarrow \infty} u_{i, n}\left(\hat{x}_{n}\right)-<p_{i}, y>-\frac{1}{2}<X_{i} y, y>-2 \delta_{i}|y|^{2} .
$$

On the other hand, by ii) in Proposition 4.10:

$$
\lim \inf _{n \rightarrow \infty} u_{i, n}\left(\hat{x}_{n}\right) \leq \lim \sup _{n \rightarrow \infty} u_{i, n}\left(\hat{x}_{n}\right) \leq v_{i}(y) .
$$

The last inequality holds because $\hat{x}_{n} \rightarrow y$ and $\hat{x}_{n} \in \Theta$, for all $n$.

Besides, since it is always true that $v(x)$ can be approximated by $v(0)$ using Taylor:

$$
v_{i}(x) \leq v_{i}(0)+<p_{i}, x>+\frac{1}{2}<X_{i} x, x>+\delta_{i}|x|^{2},
$$

for $x \in N_{r}$. If we write $y$ instead of $x$, given that $y \in N_{r}$ as well

$$
v_{i}(y) \leq v_{i}(0)+<p_{i}, y>+\frac{1}{2}<X_{i} y, y>+\delta_{i}|y|^{2},
$$

or

$$
v_{i}(y)-<p_{i}, y>-\frac{1}{2}<X_{i} y, y>-2 \delta_{i}|y|^{2} \leq v_{i}(0)-\delta_{i}|y|^{2} .
$$

Hence from (4.2) and using (4.3):

$$
\begin{gathered}
v_{i}(0) \leq \lim _{n \rightarrow \infty} \inf _{n, n}\left(\hat{x}_{n}\right)-<p_{i}, y>-\frac{1}{2}<X_{i} y, y>-2 \delta_{i}|y|^{2} \\
\leq v_{i}(y)-<p_{i}, y>-\frac{1}{2}<X_{i} y, y>-2 \delta_{i}|y|^{2} \leq v_{i}(0)-\delta_{i}|y|^{2} \\
\left.\Rightarrow v_{i}(0) \leq v_{i}(0)-\delta_{i}|y|^{2} \Rightarrow|y|^{2}=0 \Rightarrow y=0, \text { so that we have proven that } \hat{x}_{n} \rightarrow 0 . \text { By assumption } i i\right) \\
\lim \sup _{n \rightarrow \infty} u_{i, n}\left(\hat{x}_{n}\right) \leq v_{i}(0) .
\end{gathered}
$$

\footnotetext{
${ }^{4}$ Note that we have a sequence on a closed and bounded set, hence Cauchy Theorem applies.
} 
From our previous calculations in (4.2):

$$
v_{i}(0) \leq \lim \inf _{n \rightarrow \infty} u_{i, n}\left(\hat{x}_{n}\right)-<p_{i}, y>-\frac{1}{2}<X_{i} y, y>-2 \delta_{i}|y|^{2},
$$

substituting $y$ by 0 :

$$
v_{i}(0) \leq \lim \inf _{n \rightarrow \infty} u_{i, n}\left(\hat{x}_{n}\right)-<p_{i}, 0>-\frac{1}{2}<X_{i} 0,0>-2 \delta_{i}|0|^{2}=\lim \inf _{n \rightarrow \infty} u_{i, n}\left(\hat{x}_{n}\right) .
$$

Then using (4.4) and (4.5)

$$
\begin{gathered}
\lim \sup _{n \rightarrow \infty} u_{i, n}\left(\hat{x}_{n}\right) \leq v_{i}(0) \leq \lim \inf _{n \rightarrow \infty} u_{i, n}\left(\hat{x}_{n}\right) \\
\Rightarrow v_{i}(0)=\lim u_{i, n}\left(\hat{x}_{n}\right) .
\end{gathered}
$$

Next, we need to prove that

$$
\left(p_{i}+4 \delta_{i} \hat{x}_{n}+X_{i} \hat{x}_{n}, X_{i}+4 \delta_{i} I\right) \in J_{\Theta}^{2,+} u_{i, n}\left(\hat{x}_{n}\right),
$$

for every $i$ and large $n$. By definition we have that

$$
u_{i, n}(x) \leq u_{i, n}\left(\hat{x}_{n}\right)+<p_{i}+4 \delta_{i} \hat{x}_{n}+X_{i} \hat{x}_{n}, x-\hat{x}_{n}>+\frac{1}{2}<\left(X_{i}+4 \delta_{i} I\right)\left(x-\hat{x}_{n}\right), x-\hat{x}_{n}>+o\left(\left|x-\hat{x}_{n}\right|^{2}\right) .
$$

Using (4.6), we need to obtain

$$
u_{i, n}(x) \leq u_{i, n}\left(\hat{x}_{n}\right)+<p_{i}, x-\hat{x}_{n}>+\frac{1}{2}\left(<X_{i} x, x>-<X_{i} \hat{x}_{n}, \hat{x}_{n}>\right)+2 \delta\left(|x|^{2}-\left|\hat{x}_{n}\right|^{2}\right) .
$$

That is, we need to go from (4.6) to (4.7). Let us tackle term by term as they appear in (4.6). The third term on the right hand side of (4.6):

$$
\begin{aligned}
\frac{1}{2}< & \left(X_{i}+4 \delta_{i} I\right)\left(x-\hat{x}_{n}\right), x-\hat{x}_{n}>=\frac{1}{2}<X_{i}\left(x-\hat{x}_{n}\right)+4 \delta_{i} I\left(x-\hat{x}_{n}\right), x-\hat{x}_{n}> \\
= & \frac{1}{2}<X_{i}\left(x-\hat{x}_{n}\right), x-\hat{x}_{n}>+2 \delta_{i}<x-\hat{x}_{n}, x-\hat{x}_{n}> \\
= & \frac{1}{2}<X_{i} x, x>-\frac{1}{2}<X_{i} x, \hat{x}_{n}>-\frac{1}{2}<X_{i} \hat{x}_{n}, x>+\frac{1}{2}<X_{i} \hat{x}_{n}, \hat{x}_{n}> \\
& +2 \delta_{i}<x-\hat{x}_{n}, x-\hat{x}_{n}>+2 \delta_{i}|x|^{2}+2 \delta_{i}\left|\hat{x}_{n}\right|^{2}-2 \delta_{i}<x, \hat{x}_{n}>-2 \delta_{i}<\hat{x}_{n}, x>.
\end{aligned}
$$

Note that the last two terms are identical, so that we can write

$$
2 \delta_{i}<x, \hat{x}_{n}>+2 \delta_{i}<\hat{x}_{n}, x>=4 \delta_{i}<x, \hat{x}_{n}>
$$

The second term on the right hand side of (4.6):

$$
\begin{aligned}
& <p_{i}+4 \delta_{i} \hat{x}_{n}+X_{i} \hat{x}_{n}, x-\hat{x}_{n}> \\
& =<p_{i}, x-\hat{x}_{n}>+<4 \delta_{i} \hat{x}_{n}, x>-4 \delta_{i}\left|\hat{x}_{n}\right|^{2}+<X_{i} \hat{x}_{n}, x>-<X_{i} \hat{x}_{n}, \hat{x}_{n}>.
\end{aligned}
$$


Plugging them together:

$$
\begin{aligned}
< & p_{i}+4 \delta_{i} \hat{x}_{n}+X_{i} \hat{x}_{n}, x-\hat{x}_{n}>+\frac{1}{2}<\left(X_{i}+4 \delta_{i} I\right)\left(x-\hat{x}_{n}\right), x-\hat{x}_{n}> \\
= & <p_{i}, x-\hat{x}_{n}>+<4 \delta_{i} \hat{x}_{n}, x>-4 \delta_{i}\left|\hat{x}_{n}\right|^{2}+<X_{i} \hat{x}_{n}, x>-<X_{i} \hat{x}_{n}, \hat{x}_{n}> \\
& +\frac{1}{2}<X_{i} x, x>-\frac{1}{2}<X_{i} x, \hat{x}_{n}>-\frac{1}{2}<X_{i} \hat{x}_{n}, x>+\frac{1}{2}<X_{i} \hat{x}_{n}, \hat{x}_{n}> \\
& +2 \delta_{i}|x|^{2}+2 \delta_{i}\left|\hat{x}_{n}\right|^{2}-4 \delta_{i}<x, \hat{x}_{n}> \\
= & <p_{i}, x-\hat{x}_{n}>+2 \delta_{i}\left(|x|^{2}-\left|\hat{x}_{n}\right|^{2}\right)+\frac{1}{2}\left(<X_{i} x, x>-<X_{i} \hat{x}_{n}, \hat{x}_{n}>\right) \\
& +\frac{1}{2}\left(<X_{i} \hat{x}_{n}, x>-<X_{i} x, \hat{x}_{n}>\right)
\end{aligned}
$$

In order to conclude, we need to prove that for every $i$

$$
<X_{i} \hat{x}_{n}, x>\longrightarrow<X_{i} x, \hat{x}_{n}>.
$$

$X_{i}$ is a symmetric matrix so that $X_{i}^{T}=X_{i}$. Then

$$
<X_{i} \hat{x}_{n}, x>=\left(X_{i} \hat{x}_{n}\right)^{T} x=\hat{x}_{n}^{T} X_{i}^{T} x,
$$

and

$$
<X_{i} x, \hat{x}_{n}>=\left(X_{i} x\right)^{T} \hat{x}_{n}=x^{T} X_{i}^{T} \hat{x}_{n} .
$$

In particular, since all matrices $X_{i}$ are diagonal, we have that $X_{i}^{T}=X_{i}$

$$
\hat{x}_{n}^{T} X_{i} x=x^{T} X_{i} \hat{x}_{n}
$$

As a result, we have proven that inequalities (4.6) and (4.7) are identical.

Up to this point, it has been proven that one can find a sequence $\left(p_{n}, X_{n}\right)$ which converges towards $(p, X)$ in $J_{\Theta}^{2,+} u_{n}\left(\hat{x}_{n}\right)$ coordinate by coordinate, that is

$$
F(\hat{x}, u(\hat{x}), p, X) \leq 0
$$

Let us proof next Lemma 4.9.

Proof of Lemma 4.9. Suppose $z \in \Theta$ and $(p, X) \in J_{\Theta}^{2,+} w^{*}\left(\hat{x}_{n}\right)$. We need to prove that

$$
F\left(z, w^{*}(z), p, X\right) \leq 0 .
$$

Let us choose a sequence $\left(x_{n}, u_{n}\right) \in \Theta \times \mathcal{F}$ such that $\left(x_{n}, u_{n}\left(x_{n}\right)\right) \rightarrow\left(z, w^{*}(z)\right)$ and that $\lim _{\sup } \operatorname{su}_{n \rightarrow \infty} u_{n}\left(x_{n}\right) \leq$ $w^{*}(z)$, coordinate by coordinate. Then, by Proposition 4.10, $\exists\left(p_{n}, X_{n}\right) \in J_{\Theta}^{2,+} u_{n}\left(\hat{x}_{n}\right)$ for a given $\hat{x}_{n}$ and

$$
\left(\hat{x}_{n}, u_{n}\left(\hat{x}_{n}\right), p_{n}, X_{n}\right) \longrightarrow\left(z, w^{*}(z), p, X\right) .
$$

Each $u_{n}$ is a subsolution of $F$, that is,

$$
F\left(\hat{x}_{n}, u_{n}\left(\hat{x}_{n}\right), p_{n}, X_{n}\right) \leq 0 .
$$


Passing to the limit, we obtain that

$$
F\left(z, w^{*}(z), p, X\right) \leq 0 .
$$

We face now the second step in the proof of Theorem 4.5. Suppose $\Omega \subseteq \Theta \subseteq \mathbb{R}^{N}$ is open, $u$ is a solution of $F \leq 0$, and its lower hemicontinuous envelope $u_{*}$ is not a solution of $F \geq 0$. Let us remember that $u_{*}$ is defined as

$$
u_{*}(x)=\lim \inf _{r \rightarrow 0}\{u(y): y \in \Theta,|y-x|<r\} .
$$

In particular, let us assume that $0 \in \Omega$ and that $F\left(0, u_{*}(0), p, X\right)<0$ for some $(p, X) \in J_{\Omega}^{2} u_{*}(0)$. Let us build an approximation to $u_{*}(0)$ choosing small and positive constants $\gamma_{i}, \delta_{i} \in \mathbb{R}$ such that

$$
u_{i, \delta_{i}, \gamma_{i}}(x)=u_{* i}(0)+\delta+<p_{i}, x>+\frac{1}{2}<X_{i} x, x>-\gamma_{i}|x|^{2} .
$$

By continuity, $u_{\delta, \gamma}=\left(u_{1, \delta_{1}, \gamma_{1}}, \ldots, u_{N, \delta_{N}, \gamma_{N}}\right)$ is a solution of $F \leq 0$ in $B_{r}=\{x:|x|<r\}$ for small and positive $r, \delta_{i}, \gamma_{i} \in \mathbb{R}$.

Theorem 4.5 ensures that the supremum of the subsolutions is a solution. The theorem will be proven by contradiction. Suppose that the supremum is not a solution, or that there exists a point at which it is not. Let us take for instance $0 \in \Omega$ and assume that $\exists j \in\{1, \ldots, N\}$ such that $F_{j}\left(0, u_{*}(0), p, X\right)<0$.

Invoking continuity again, we can build a subsolution $u_{\delta, \gamma}$ which is close to $u_{*}(0)$. That is $u_{\delta, \gamma}$ would need to verify that

$$
u_{i, \delta_{i}, \gamma_{i}}(x) \geq u_{* i}(x)=u_{* i}(0)+<p_{i}, x>+\frac{1}{2}<X_{i} x, x>+o\left(|x|^{2}\right),
$$

for all $i$. The inequality in (4.9) is true because $u_{*}=\liminf \{u(y):|y-r|<r\}$. Then we apply Taylor's approximation of $u_{* i}$ around 0 .

Let us choose $\delta_{i}=\left(\frac{r^{2}}{8}\right)^{\gamma}$ for all $i$, then $u(x)=u_{\delta, \gamma}(x)$ for $\frac{r}{2}<|x|<r$, if $r$ is sufficiently small.

Next, let us define $U(x)=\left(U_{1}(x), \ldots, U_{N}(x)\right)$ as

$$
U_{i}(x)=\left\{\begin{array}{l}
\max \left\{u_{i}(x), u_{i, \delta_{i}, \gamma_{i}}(x)\right\}, \text { if }|x|<r \\
u_{i}(x) \text { otherwise. }
\end{array}\right.
$$

By Lemma 4.9, since $u_{\delta, \gamma}$ is a solution of $F \leq 0$ and bounded, then $U(x)$ is a solution of $F \leq 0$ in $\Omega$.

Note that in every neighborhood of 0 , there are points such that $U(x) \geq u(x)$, i.e. $U_{i}(x) \geq u_{i}(x)$ for $i=$ $1, \ldots, N$. Indeed, we can build a sequence $\left(x_{n}, u_{n}\left(x_{n}\right)\right)$ convergent to $\left(0, u_{*}(0)\right)$ and then

$$
\lim _{n \rightarrow \infty}\left(U_{i}\left(x_{n}\right)-u_{i}\left(x_{n}\right)\right)=u_{i, \delta_{i}, \gamma_{i}}(0)-u_{*, i}(0)=u_{*, i}(0)+\delta_{i}-u_{*, i}(0)=\delta_{i}>0 .
$$

We have proven the following lemma:

Lemma 4.11. Let $\Omega$ be open and $u$ a solution of $F \leq 0$ in $\Omega$. If $u_{*}$ fails to be a supersolution at some point $z$, i.e. there exists $(p, X) \in J_{\Omega}^{2,-} u_{*}(z)$, for which $F\left(z, u_{*}(z), p, X\right)<0$, then for any small $\kappa>0$, there is a 
subsolution $U_{\kappa}$ of $F \leq 0$ in $\Omega$ satisfying

$$
\begin{aligned}
& U_{\kappa}(x) \geq u(x) \text { and } \sup \left(U_{\kappa}-u\right)>0, \\
& U_{\kappa}(x)=u(x) \text { for } x \in \Omega,|x-z|>\kappa .
\end{aligned}
$$

We are ready now to prove Theorem 4.5 , i.e. that

$$
\underline{u}_{*} \leq W_{*} \leq W \leq W^{*} \leq \bar{u}^{*}
$$

and in particular

$$
W_{*}=W=W^{*}=0, \text { on } \delta \Omega .
$$

Proof of Theorem 4.5. By Lemma 4.9, $W^{*}$ is a subsolution of $D P$ and by comparison $W^{*} \leq \bar{u}$, where $\bar{u}$ is a supersolution (and a subsolution is smaller than a supersolution). By the definition of $W$ :

$$
W(x)=\sup \{w(x): \underline{u} \leq w \leq \bar{u} \text { and } w \text { is a subsolution of } \mathrm{DP}\}
$$

$W$ is a subsolution, so that $W=W^{*}$.

Then, if $W_{*}$ was a supersolution, then the proof would be completed, because $W_{*} \geq W^{*}$. On the one hand because $W_{*}$ is a supersolution. On the other hand, by construction, $W_{*} \leq W^{*} \Rightarrow W_{*}=W^{*}=W$.

Suppose that $W_{*}$ is not a supersolution at some point $z \in \Omega$. Then we are in the premises of Lemma 4.11, and it will be because $\exists j \in\{1, \ldots, N\}$ such that

$$
F_{j}(z, u(z), p, X)<0
$$

Then, we invoke Lemma 4.11 and build a function $W_{\kappa}$ such that

$$
\begin{aligned}
& W_{\kappa}(x) \geq W_{*}(x) \text { and } \sup _{\Omega}\left(W_{\kappa}-W_{*}\right) \geq 0, \\
& W_{\kappa}(x)=W_{*}(x), \text { for } x \in \Omega,|x-z|>\kappa .
\end{aligned}
$$

Since $W_{\kappa}$ is a subsolution, $\underline{u} \leq W_{\kappa}$, and $W_{\kappa}=0$ on $\delta \Omega$. By comparison, since $W_{\kappa}$ is a subsolution, $W_{\kappa} \leq \bar{u}$. Since $W$ is the maximal subsolution between $\underline{u}$ and $\bar{u} \Rightarrow W_{\kappa} \leq W$, which is a contradiction. Then, $W_{*}$ is a supersolution.

\subsection{An upper and a lower solution}

In this section, we find suitable upper and lower solutions to $O$ under given assumptions regarding the parameter set and functional forms. First, we assume that technology is spatially homogeneous so that $A_{x}(x, t)=$ 0 for all $(x, t)$. Second, utility is measured via a CIES utility function with parameter $\sigma$, i.e. $u(c)=\frac{c^{1-\sigma}}{1-\sigma}$. As a result, consumption per capita is $c=\left(A h^{\alpha-1} r^{1-\alpha}\right)^{-\sigma}$. By definition we say that $(\underline{\mathrm{h}}, \underline{\mathrm{r}})$ is a lower solution to our problem if

$$
L S\left\{\begin{array}{l}
\underline{\mathrm{h}}_{t}-\underline{\mathrm{h}}_{x x}+A^{1-\alpha}(1-\sigma) \frac{\underline{\mathrm{r}}^{(1-\alpha)(1-\sigma)}}{\underline{\mathrm{h}}^{1+(1-\alpha)(1-\sigma)}} \underline{\mathrm{h}}_{x} \leq 0 \\
\underline{\mathrm{r}}_{t}-\delta A^{-\sigma}\left(\frac{\overline{\mathrm{h}}}{\underline{\underline{\mathrm{h}}}}\right)^{(1-\alpha) \sigma} \leq 0
\end{array}\right.
$$


Let us define $\underline{\mathrm{h}}=h_{0}-H t$, and $\underline{\mathrm{r}}=R$, where $R \in \mathbb{R}$ is a real constant small enough and it will be properly constrained later on. Regarding $H$, we require that

$$
\left\{\begin{array}{l}
H \geq \sup _{x}\left|h_{0, t}-h_{0, x x}+A^{1-\alpha}(1-\sigma) \frac{r_{0}^{(1-\alpha)(1-\sigma)}}{h_{0}^{1+(1-\alpha)(1-\sigma)}} h_{0, x}\right| \\
H T \leq \inf _{x} h_{0}
\end{array}\right.
$$

where $T$ is the time horizon. Next, we prove that $(\underline{\mathrm{h}}, \underline{\mathrm{r}})=\left(h_{0}-H t, R\right)$ is a lower solution. Replacing $(\underline{\mathrm{h}}, \underline{\mathrm{r}})$ in the first equation of LS by $\left(h_{0}-H t, R\right)$, we obtain that

$$
\begin{aligned}
& -H-h_{0, x x}+A^{1-\sigma}(1-\alpha) \frac{R^{(1-\alpha)(1-\sigma)}}{\left(h_{0}-H t\right)^{1+(1-\alpha)(1-\sigma)}} h_{0, x} \\
& \leq h_{0, x x}-A^{1-\sigma}(1-\alpha) \frac{r_{0}^{(1-\alpha)(1-\sigma)}}{h_{0}^{1+(1-\alpha)(1-\sigma)}} h_{0, x}-h_{0, x x}+A^{1-\sigma}(1-\alpha) \frac{R^{(1-\alpha)(1-\sigma)}}{\left(h_{0}-H t\right)^{1+(1-\alpha)(1-\sigma)}} h_{0, x} \\
& =-A^{1-\sigma}(1-\alpha) h_{0, x}\left(\frac{r_{0}^{(1-\alpha)(1-\sigma)}}{h_{0}^{1+(1-\alpha)(1-\sigma)}}-\frac{R^{(1-\alpha)(1-\sigma)}}{\left(h_{0}-H t\right)^{1+(1-\alpha)(1-\sigma)}}\right),
\end{aligned}
$$

which is negative under the assumptions we have made for $H$, whenever $h_{0, x}$ is positive and

$$
R^{(1-\alpha)(1-\sigma)} \leq \inf _{x}\left(h_{0}-H T\right) \inf _{x} \frac{r_{0}^{(1-\alpha)(1-\sigma)}}{h_{0}^{1+(1-\alpha)(1-\sigma)}}
$$

The second equation in $L S$ trivially holds for $(\underline{\mathrm{h}}, \underline{\mathrm{r}})$ since $\underline{\mathrm{r}}_{t}=0$ and both $\underline{\mathrm{h}}$ and $\underline{\mathrm{r}}$ are positive. We turn our attention to the existence of an upper solution. A couple $(\bar{h}, \bar{r})$ is an upper solution if

$$
U S\left\{\begin{array}{l}
\bar{h}_{t}-\bar{h}_{x x}+A^{1-\sigma}(1-\alpha) \frac{\bar{r}^{(1-\alpha)(1-\sigma)}}{h^{1+(1-\alpha)(1-\sigma)}} \bar{h}_{x} \geq 0 \\
\bar{r}_{t}-\delta A^{-\sigma}\left(\frac{\bar{h}}{\bar{r}}\right)^{(1-\alpha) \sigma} \geq 0
\end{array}\right.
$$

Let us fix $(\bar{h}, \bar{r})=\left(h_{0}+H t, R+\Gamma t\right)$. The second equation in $U S$ is satisfied whenever

$$
\Gamma-\delta A^{-\sigma}\left(\frac{h_{0}+H t}{R+\Gamma t}\right)^{(1-\alpha) \sigma} \geq 0
$$

or

$$
\Gamma \geq \sup _{x, t}\left\{\delta A^{-\sigma}\left(\frac{h_{0}+H t}{R+\Gamma t}\right)^{(1-\alpha) \sigma}\right\}=\sup _{x}\left\{\delta A^{-\sigma}\left(\frac{h_{0}+H T}{R}\right)^{(1-\alpha) \sigma}\right\} .
$$

Regarding the first equation, our upper solution needs to verify then that

$$
H-h_{0, x x}+A^{1-\sigma}(1-\alpha) \frac{(R+\Gamma t)^{(1-\alpha)(1-\sigma)}}{\left(h_{0}+H t\right)^{1+(1-\alpha)(1-\sigma)}} \bar{h}_{0, x} \geq 0 .
$$


We know that

$$
\begin{aligned}
& H-h_{0, x x}+A^{1-\sigma}(1-\alpha) \frac{(R+\Gamma t)^{(1-\alpha)(1-\sigma)}}{\left(h_{0}+H t\right)^{1+(1-\alpha)(1-\sigma)}} \bar{h}_{0, x} \\
& \geq h_{0, x x}-A^{1-\sigma}(1-\alpha) \frac{r_{0}^{(1-\alpha)(1-\sigma)}}{h_{0}^{1+(1-\alpha)(1-\sigma)}} \bar{h}_{0, x}-h_{0, x x}+A^{1-\sigma}(1-\alpha) \frac{(R+\Gamma t)^{(1-\alpha)(1-\sigma)}}{\left(h_{0}+H t\right)^{1+(1-\alpha)(1-\sigma)}} \bar{h}_{0, x} \\
& =A^{1-\sigma}(1-\alpha) \bar{h}_{0, x}\left(\frac{r_{0}^{(1-\alpha)(1-\sigma)}}{h_{0}^{1+(1-\alpha)(1-\sigma)}}+\frac{(R+\Gamma t)^{(1-\alpha)(1-\sigma)}}{\left(h_{0}+H t\right)^{1+(1-\alpha)(1-\sigma)}}\right),
\end{aligned}
$$

which is positive if $h_{0, x} \geq 0$.

\section{NuMERICAL EXERCISES}

We come back to $O$, our motivating problem. In this section we illustrate numerically some of $O$ 's spatialdynamic properties. First we explore the importance of initial endowments, both in terms of labor and the environmental quality. Summarizing our results, we find on the one hand that initial differences in the distribution of population fade away with time. Indeed, since individuals move following the gradient of their welfare, they will move towards areas providing higher welfare at a given moment in time. Migration smoothes out spatial differences. However, on the other hand, we find that if the environmental quality is not evenly distributed it can trigger everlasting inequalities. Lasting inequalities stem from the non-mobility of the environmental quality. These two first exercises consider three different levels of diffusion. Our results show that diffusion does not play a major qualitative role. Instead, its role is mainly quantitative, accelerating or decelerating the dynamics of population.

Our third and fourth exercises analyse the roles of heterogeneity in total factor productivity and in environmental sensitivity. These simulations illustrate the existence of opposite migration forces. We distinguish between primary forces that privilege the agglomeration of individuals to increase production, and secondary forces that affect individuals utility in various ways, such as technological disparities.

Finally, our last simulation examines the dynamics of an economy in which individuals' welfare depends not only on consumption but also on the environmental quality.

In order to simulate our model, we consider a standard discretization of $O$. Space is the interval $[0,5]$ and the time span is $[0,60]$. Regarding the choice of our functional forms, we consider that the production of the unique good is described by a Cobb-Douglas function:

$$
Y(h, r)=A h^{\alpha} r^{1-\alpha},
$$

where $A$ stands for total factor productivity and $\alpha \in(0,1)$ is the share of labor in production. In all exercises but in 5.3, $A$ is homogeneous in time and in space. In particular, we assume there that $A \equiv 1$.

We assume a constant elasticity of substitution utility function

$$
u(c)=\frac{c^{1-\sigma}}{1-\sigma},
$$

where $\sigma \in \mathbb{R}$. From Section 4.3, we know that problem $O$ endowed with the production and utility functions as described above verifies the premises of Theorem 4.5. As a consequence, we can ensure the existence of a solution to our problem.

In the first two exercises we inspect three different degrees of diffusion $d, d \in\{0.0075,0.015,0.5\}$. The benchmark level of population mobility across locations is represented by the intermediate value for $d, d=0.015$. Setting tighter barriers to migration will divide this coefficient by half, i.e., $d=0.0075$. Finally, the case $d=0.5$ stands for a high degree of mobility in which half of the individuals willing to migrate succeed. Table 1 assembles the values adopted in our simulations for the other parameters. 
TABLE 1. Parameters values.

\begin{tabular}{lll}
\hline$\sigma$ & Utility parameter & 0.75 \\
$\gamma$ & Regeneration parameter & 0.008 \\
$\delta$ & Damage parameter & 0.02 \\
$\alpha$ & Output elasticity & 0.75 \\
\hline
\end{tabular}
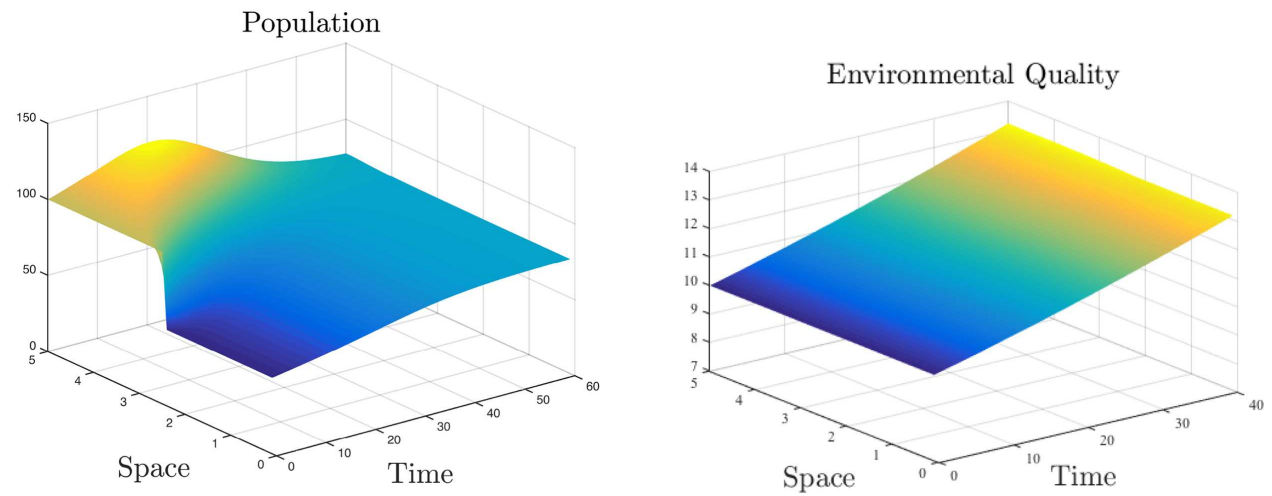

Figure 1. Heterogeneous $h(x, 0)$. Benchmark case.
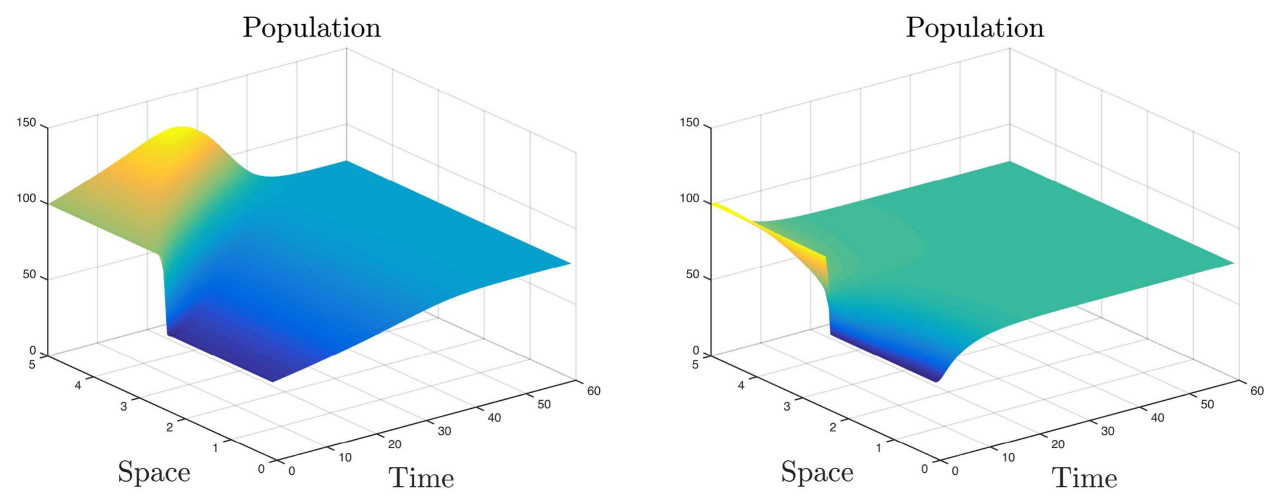

FiguRE 2. Heterogeneous $h(x, 0)$. Left: $d=0.0075$. Right: $d=0.5$.

\subsection{Heterogenous initial population}

Let us first study the case of an economy where the initial population is not uniformly distributed across locations. In particular, we assume that the first half of space is less populated than the second half:

$$
h(x, 0)=\left\{\begin{array}{l}
50, \text { if } x \in[0,2.5), \\
100, \text { if } x \in[2.5,5] .
\end{array}\right.
$$

Note that in this first exercise the only source of spatial heterogeneity is the initial distribution of the population. Our results are displayed in Figures 1 and 2.

Figure 1 considers the intermediate level of population mobility $(d=0.015)$. The diffusion mechanism due to utility differentials across locations tends to homogenize both population and environmental quality. This is particularly evident when one examines the effect of the degree of population mobility. Figure 2 compares the situations of low (on left hand side) and high (on the right hand side) mobility. In contrast to Figure 1, greater 

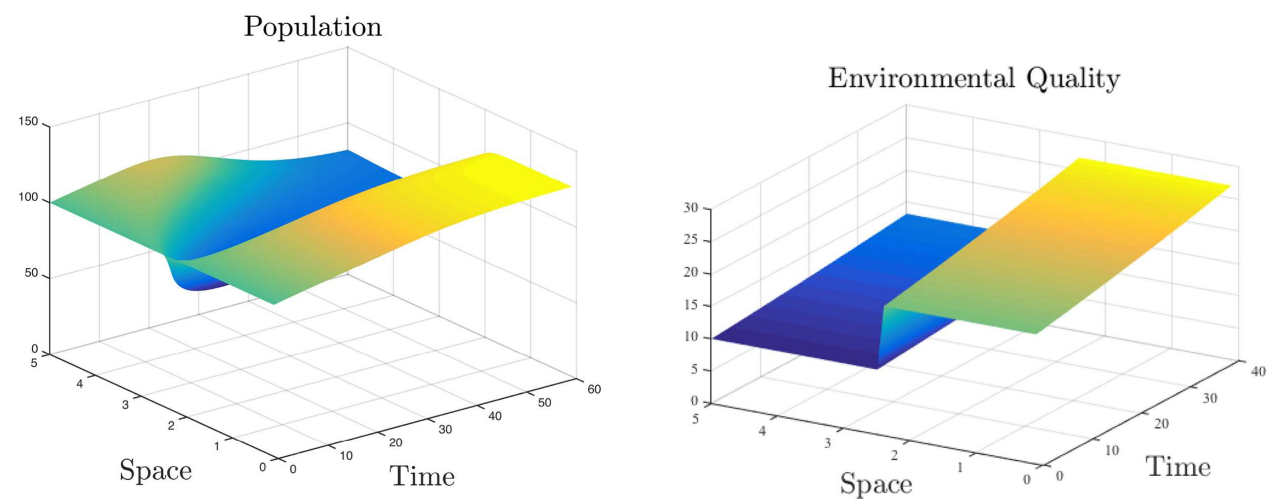

FiguRE 3. Heterogeneous $r(x, 0)$. Benchmark case.
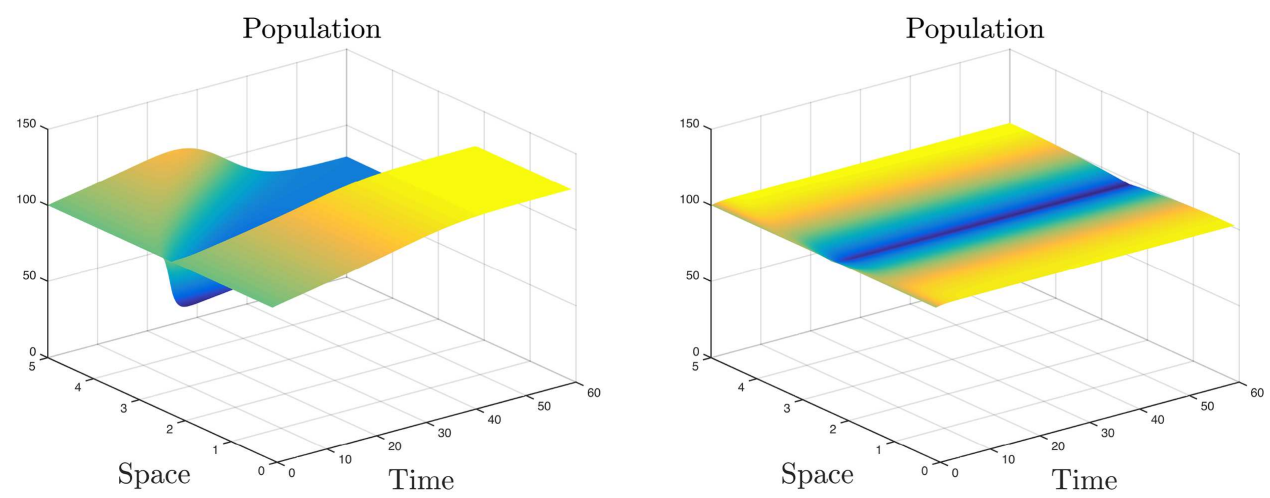

FiguRE 4. Heterogeneous $r(x, 0)$. Left: $d=0.0075$. Right: $d=0.5$.

mobility of population reduces transitional spatial disparities. However, barriers to migration significantly foster spatial inequalities.

\subsection{Heterogenous initial environmental quality}

In order to further investigate the role played by spatial mobility, we study the case of an initial unequal distribution of the environmental quality, which represents the unique source of spatial heterogeneity in this exercise:

$$
r(x, 0)= \begin{cases}20, & \text { if } x \in[0,2.5), \\ 10, & \text { if } x \in[2.5,5] .\end{cases}
$$

Figure 3 describes the evolution of population and environmental quality in the benchmark scenario. Notice how the spatial inequality of resources increases with time. The inequality in environmental quality nurtures population asymmetries across locations. The persistence of spatial asymmetries is consistent with the formation of spatial patterns. As Smith et al. [35] and Camacho and Pérez-Barahona [12] point out, the underlying mechanism is a "flux equilibrium", where the diffusion flux can maintain a spatially heterogenous equilibrium. As in the previous section, diffusion forces play an important role to reduce spatial population heterogeneity. Our simulations show that facilitating population mobility significantly reduces spatial disparity in population (see Fig. 4). Nevertheless, the evolution of the environmental quality is similar to Figure 3. Initial disparities in 

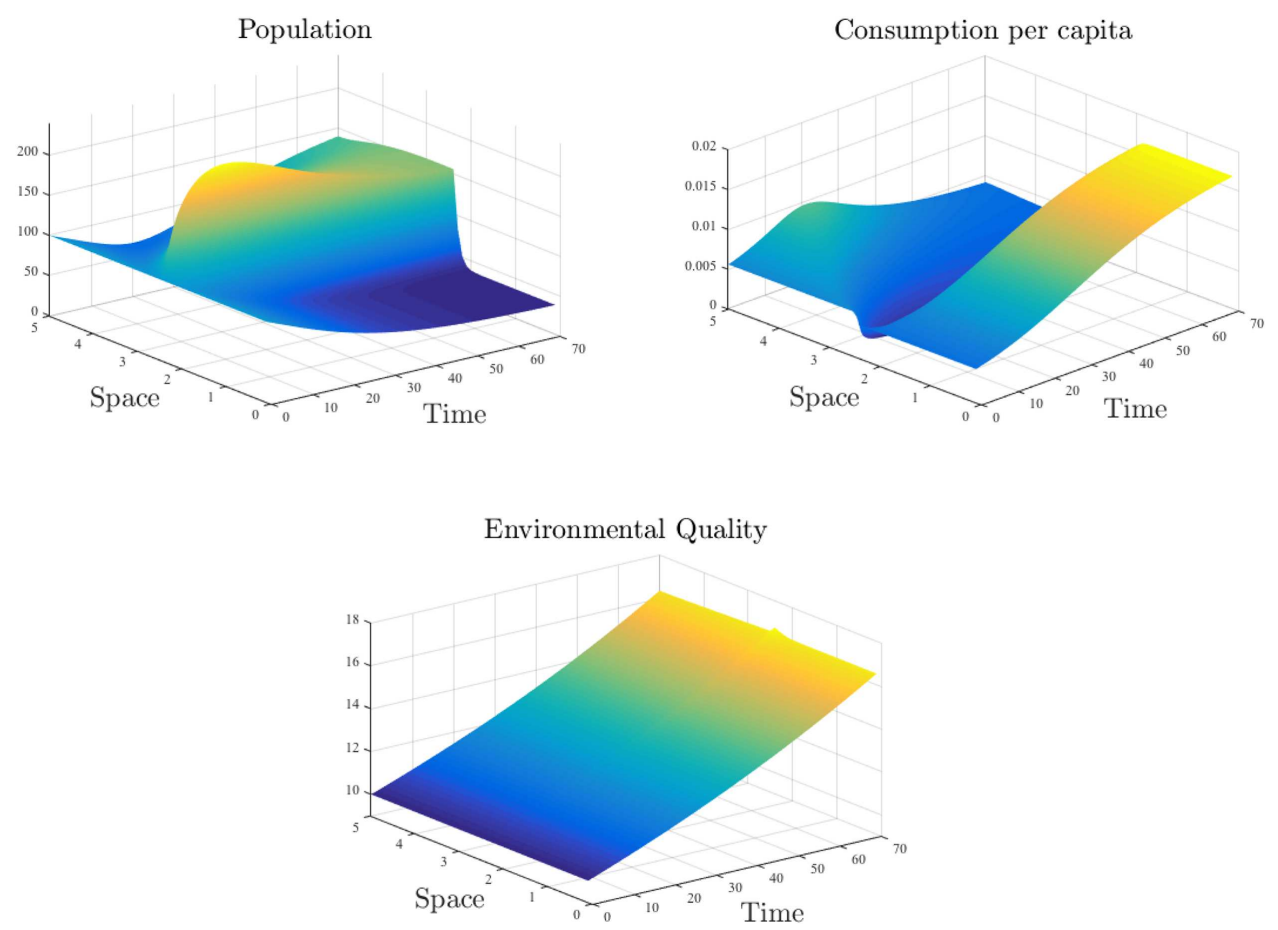

Figure 5. Permanent technological gap.

the environmental quality do not disappear with time but they accrue instead. This actually true even under situations of a high degree diffusion.

\subsection{Heterogenous technology}

In order to investigate the impact of technological spatial differences in the economy we modify parameter $A$, which represents the total factor productivity of each location. We divide the geographical space in two halves. The total factor productivity of locations in the first half of the space is $75 \%$ of the level of this technological parameter for locations in the second half:

$$
A(x, t)=\left\{\begin{array}{l}
0.75, \text { if } x \in[0,2.5) \\
1, \quad \text { if } x \in[2.5,5]
\end{array}\right.
$$

for every $t$. Results are displayed in Figure 5 .

At the beginning of the simulation individuals agglomerate around the spatial center. We clearly observe a confrontation of opposite migration forces. On the one hand, there is the technological gap force that would induce individuals to move towards the technologically advanced region in order to benefit from higher consumption. On the other hand, there are geographical primary forces. As a matter of fact production can also increase by accumulating labor for a given technological level. For our parametrization geographical forces prevail. Then, population agglomerates in the geographical center. Since our individuals are myopic and non-coordinated, they generate a drop in consumption per capita. After this transitional phase, the technology gap motive overcomes the agglomeration force and individuals start moving towards the technologically most advanced region.

Although not obvious from the population picture in Figure 5, the initial massive outflow of population relatively empties the left border. As a consequence, we observe the temporal emergence of a small region in 

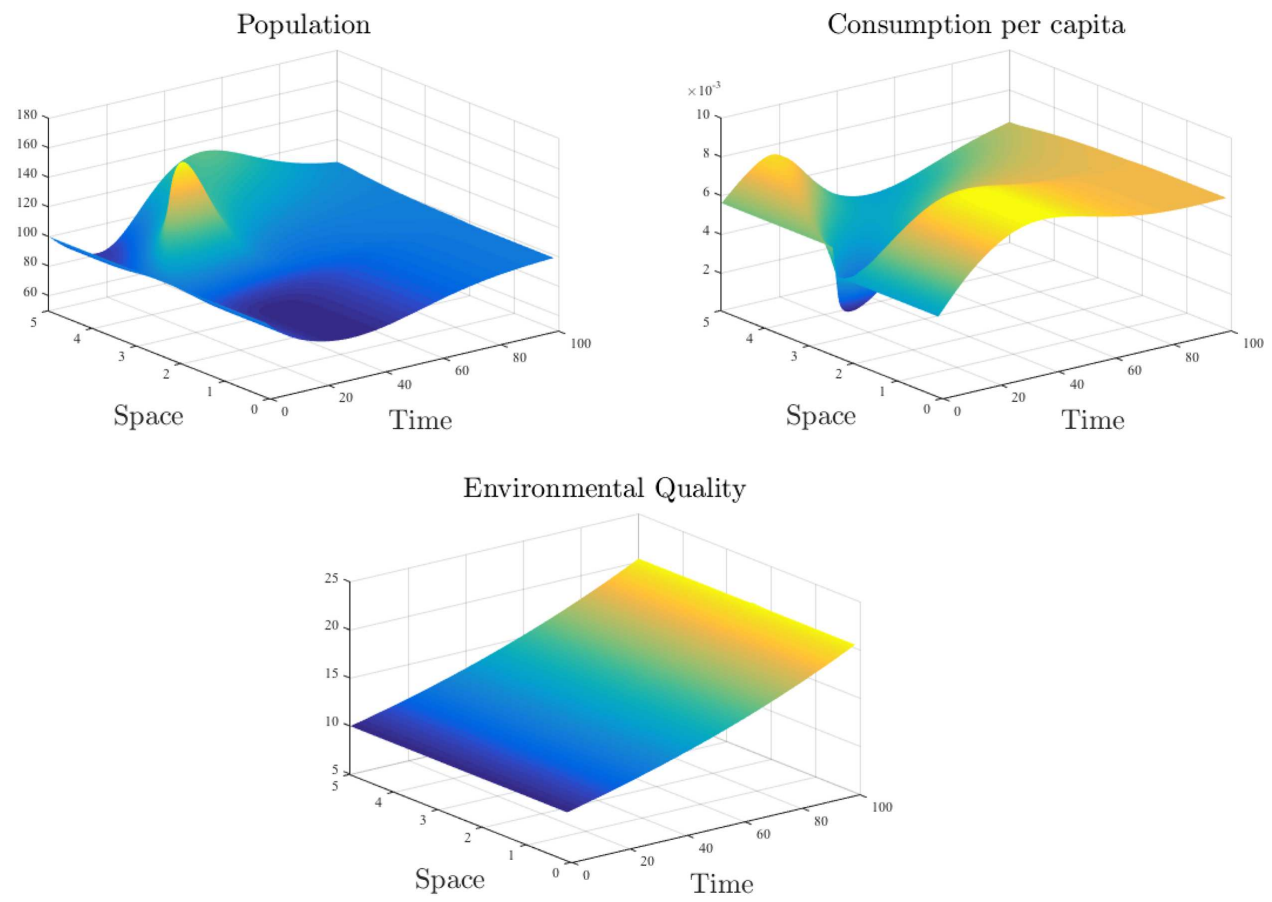

FiguRE 6. Temporary technological gap.

that border, which offers the maximum level of consumption per capita in the entire economy. Once this region emerges, individuals move in to benefit from this enhanced consumption. As a result, the advantage smoothes out with time.

In the long-run locations with the better technology attract most of population. Indeed, there is an upward jump in population from the least to the most advance region as from $t=50$. A permanent technological gap induces permanent inequalities in population distribution. Notice that inside each region the distribution is not uniform either. Population is higher the closer we get to the center. Individuals still seek to agglomerate in order to increase production, moving towards the center. Let us finally underline that consumption per capita is higher in the region endowed with the less performing technology.

Next, we study the case where the technological gap is not permanent. In this scenario the technologically backward region catches up with the most advanced region:

$$
A(x, t)=\left\{\begin{array}{l}
1-0.25 e^{-0.005 t}, \text { if } x \in[0,2.5) \\
1, \text { if } x \in[2.5,5]
\end{array}\right.
$$

for every $t$. At the beginning the trajectories for population and the environmental quality are qualitatively similar to the case of permanent technological gap (see Fig. 6). That is, individuals start by agglomerating around the geographical center. The agglomeration attains its maximum population at $t=5$, losing population from that moment onwards. The agglomeration lasts less than when the shock was permanent. From $t=5$ individuals move towards the technologically more advanced locations. At $t=50$, we find that population attains a maximum at the left border and it decreases smoothly towards the right border. As time passes, technology equalizes across locations and population starts moving back towards the right, once the advantage of the far-left disappears. 

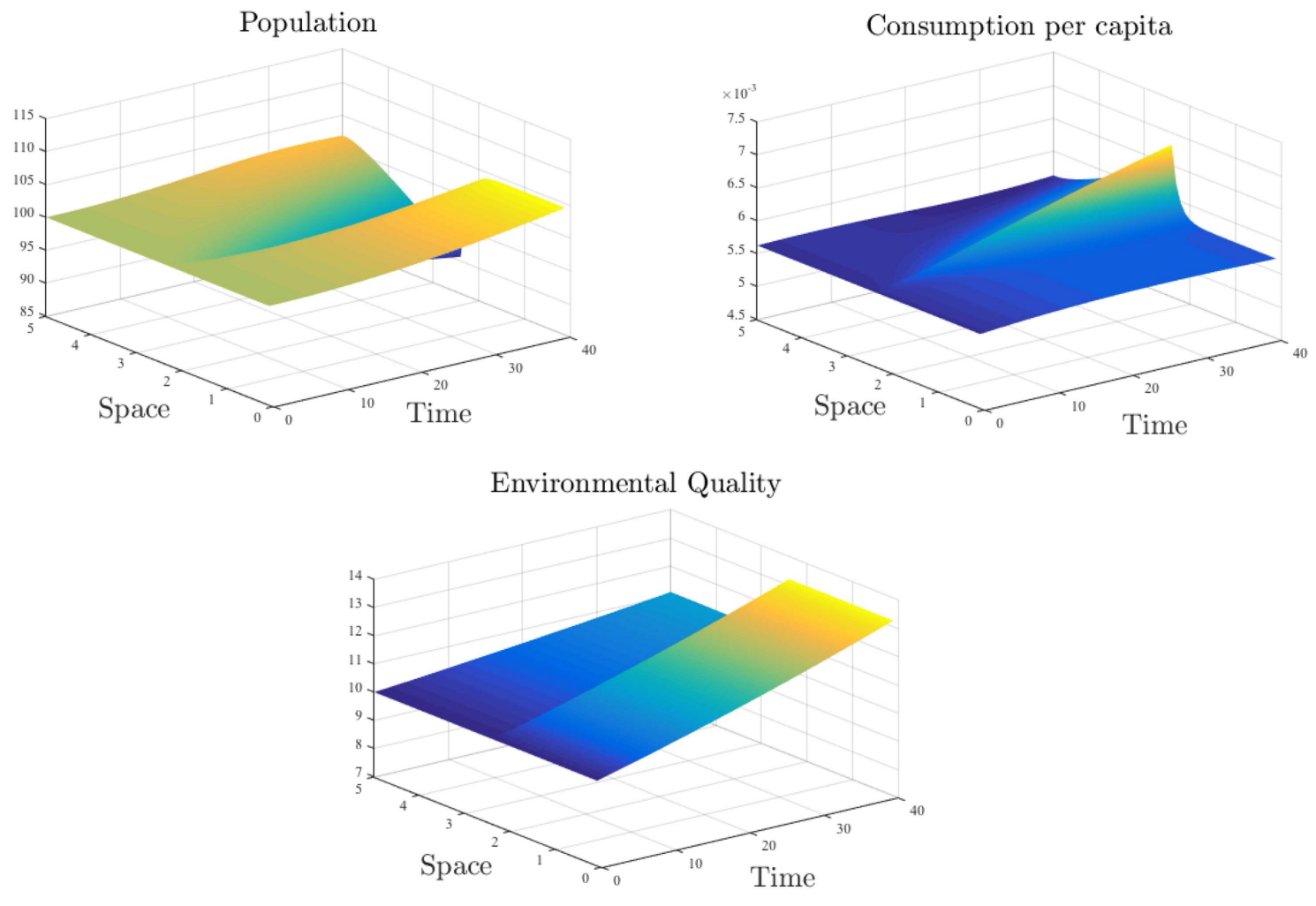

FigURE 7. Heterogeneous sensitivity to pollution.

\subsection{Heterogenous environmental sensitivity, $\delta$}

Here, we analyse the case of an economy in which the environment reacts differently to production. The effect of production on the environmental quality is captured in our model by parameter $\delta$, which becomes spatially heterogeneous as follows:

$$
\delta(x, 0)=\left\{\begin{array}{l}
0.02, \text { if } x \in[0,2.5), \\
0.1, \text { if } x \in[2.5,5]
\end{array}\right.
$$

Results are displayed in Figure 7. As in the previous exercise, we also identify the confrontation of opposite migration forces: the usual primary geographical force and a secondary force represented by the spatial heterogeneity of the environmental sensitivity. As it is clear from Figure 7 population does not agglomerate in the region with the highest environmental quality. Then, in contrast to the previous simulation, we can conclude that both migration forces prevail.

\subsection{Including resources in the utility function}

Let us assume that the utility function depends on consumption and on environmental quality according to the following law:

$$
u(c, r)=\frac{c^{1-\sigma}}{1-\sigma} \frac{r^{1-\xi}}{1-\xi}
$$



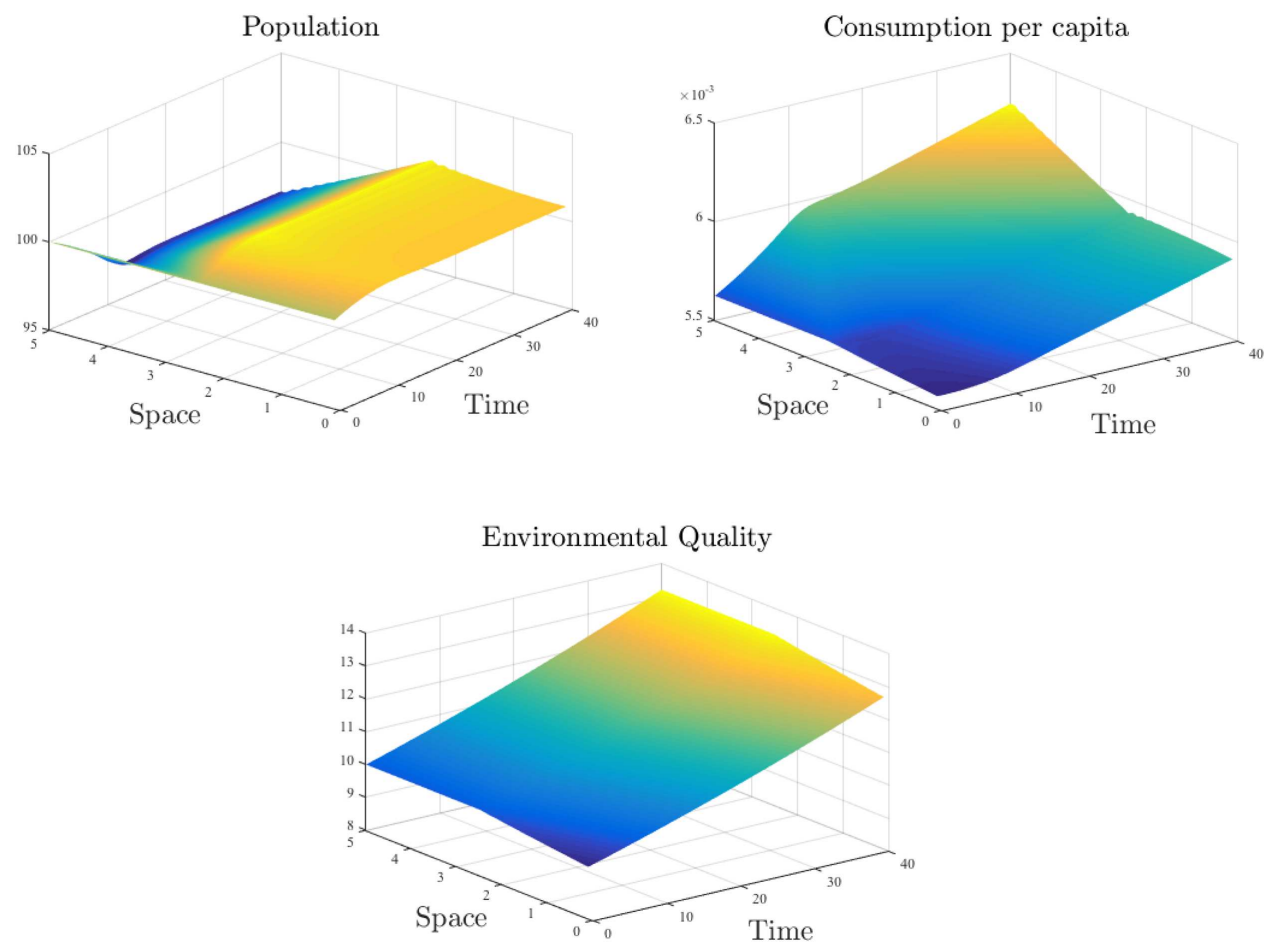

FiguRE 8. Resource dependent utility. Heterogenous continuous initial resource.

where $\xi=0.0005$. We assume that initially population is uniformly distributed across space, namely that $h(x, 0)=100$. However, environmental quality is unequally distributed in a continuous manner:

$$
r(x, 0)=\left\{\begin{array}{l}
8+2 e^{0.01(x-2.5)}, \text { if } x \in[0,2.5), \\
10, \text { if } x \in[2.5,5]
\end{array}\right.
$$

Figure 8 describes our results. Let us underline several novel features that this exercise reveals. Our exercise lets us observe that when individuals care about the environmental quality, they tend to preserve the environment. Surprisingly, without any policy maker or environmental recommendation, when individuals care about the environmental quality, they restrict their migratory flow towards better endowed locations. The reason behind is the interaction between the individuals' myopia regarding the spatial consequences of their actions and the substitutable between consumption and environmental quality.

Notice finally that, in line with Volpert et al. [39], migration waves emerge in the long-run. We understand waves as the final result of the confrontation of the migration forces. ${ }^{5}$

\section{Conclusions}

In this paper, we have built a model, which explains migration as founded on welfare differentials. Here, individuals react to the gradient of their indirect utility rather than to the gradient of salaries as in the original

\footnotetext{
${ }^{5}$ If the environmental quality is initially unequally distributed but with a step, the economy develops travelling waves in the medium term. These waves' length increases fast with time and the solution degenerates becoming unfeasible. Therefore, in order to obtain sensitive results we introduce heterogeneity in the environmental quality in a smooth manner. For similar reasons, we need to recalibrate the utility function. Indeed, keeping $\sigma=0.75$ generates unstable waves, and this for all parameter configurations we have tried. For this reason, we have set $\sigma=0.5$ in this exercise.
} 
law. Under standard assumptions on the utility function, we can prove the existence of solution for each initial distribution of human capital and natural resources. We have analyzed the corresponding dynamics of human capital and environmental quality. In particular, we numerically find that barriers to migration significantly shape the geography of the economy.

The framework constitutes a first stone of a more sophisticated world description. A possible extension could also allow for mobility of natural resources. Previous papers have pointed out role played by the spread of pollutants or fishery resources to explain many spatial patterns observed in reality. Based on the spatial generalization of the Ramsey model (see, for instance, [8]), Camacho and Perez-Barahona [12] provide a spatialdynamic framework to study the optimal use of land, encompassing land-use activities and environmental degradation. They focus on the spatial externalities of land-use management as drivers of spatial patterns: land is immobile by nature, but local actions affect the whole space since pollution flows across locations resulting in environmental damages. This stylized model manages to reproduce a great variety of spatial patterns related to the interaction between land use activities and the environment. More specifically, in line with Costello and Polasky [13], they show that the spatial connectivity together with the particular characteristics of each location can economically rationalize the creation of temporal or permanent natural reserves. A future direction to follow could include the interaction between population movements and the spatial distribution of natural resources. We also leave for further research the question of optimal population dynamics. In contrast to our simple set-up, this would require to consider a policy maker that maximizes aggregated welfare. She would take into account the consequences that her choices will have on the dynamic evolution of human, physical and environmental capital.

\section{REFERENCES}

[1] A.A. Afolayan and I.O. Adelekan, The role of climatic variations on migration and human health in Africa. Environmentalist 18 (1999) 213-318.

[2] C. Aliprantis and K. Border, Infinite dimensional analysis. Springer, 2006.

[3] F.J. Alvarez and P. Mossay, Estimation of a continuous spatio-temporal population model. J. Geogr. Syst. 8 (2006) $307-316$.

[4] M.J. Beckmann and T. Puu, Spatial economics: density, potential, and flow. North-Holland (1985).

[5] M.J. Beckmann, On spatial population dynamics. Chaos Solitons Fract. 18 (2003) 439-444.

[6] M. Beine, F. Docquier and H. Rapoport, Brain drain and economic growth: theory and evidence. J. Dev. Econ. 64 (2001) $275-289$.

[7] G.J. Borjas, The labor demand curve is downward sloping: reexamining the impact of immigration on the labor market. Quart. J. Econ. 118 (2003) 1335-1374.

[8] R. Boucekkine, C. Camacho and B. Zou, Bridging the gap between growth theory and the new economic geography: the spatial Ramsey model. Macroecon. Dyn. 13 (2009) 20-45.

[9] R. Boucekkine, G. Fabbri, F. Salvatore and F. Gozzi, Growth and agglomeration in the heterogeneous space: a generalized AK approach. J. Econ. Geogr. 39 (2018) 1001-1024.

[10] C. Camacho, F. Mariani and L. Pensieroso, Illegal immigration and the shadow economy. Int. Tax Public Finance 24 (2017) 1050-1080.

[11] C. Camacho, F. Mariani and L. Pensieroso, Dealing with illegal immigration: the role of informality, taxation and trade. Econ. Ital. 2018 (2018) 97-122.

[12] C. Camacho and A. Pérez-Barahona, Land use change and the environment: a spatial dynamics approach. J. Econ. Dyn. Cont. 52 (2015) 96-118.

[13] C. Costello and S. Polasky, Optimal harvesting of stochastic spatial resources. J. Environ. Econ. Manag. 56 (2008) 1-18.

[14] M.G. Crandall, H. Ishii and P.L. Lions, User's guide to viscosity solutions of second order partial differential equations. Bull. Am. Math. Soc. 27 (1992) 1-67.

[15] M. Fujita, P. Krugman and A. Venables, The Spatial Economy. Cities, Regions and International Trade. MIT Press (1999).

[16] M. Fujita and T. Mori, Frontiers of the new economic geography. Papers Regl. Sci. 84 (2005) 301-521.

[17] M. Fujita and J.F. Thisse, Economics of Agglomeration. Cambridge University Press (2002).

[18] K. Fukao and R. Benabou, History versus expectations: a comment. Quart. J. Econ. 108 (1993) 535-542.

[19] M. Gianetti, On the mechanics of migration decisions: skill complementarities and endogenous price differentials. J. Dev. Econ. 71 (2003) 329-349.

[20] S. Henry, P. Boyle and E. Lambin, Modelling inter-provincial migration in Burkina Faso, West Africa: the role of sociodemographic and environmental factors. Appl. Geogr. 23 (2003) 115-136.

[21] H. Hotelling, Stability in competition. Econ. J. 39 (1929) 41-57.

[22] P. Krugman, Increasing returns and economic geography. J. Polit. Econ. 99 (1991) 483-499.

[23] P. Krugman, The Self-Organizing Economy, Appendix: A Central Place Model, Blackwell, Cambridge, MA (1996). 
[24] L. Marchiori, J. Maystadt and I. Schumacher, The impact of weather anomalies on migration in sub-Saharan Africa. J. Environ. Econ. Manag. 63 (2012) 355-374.

[25] F. Mariani, A. Pérez-Barahona and N. Raffin, Life expectancy and the environment. J. Econ. Dyn. Cont. 34 (2010) $798-815$.

[26] P. Mossay, Increasing returns and heterogeneity in a spatial economy. Regl. Sci. Urban Econ. 33 (2003) $419-444$.

[27] P. Mossay, A theory of rational spatial agglomerations. Regl. Sci. Urban Econ. 43 (2013) 385-394.

[28] N. Myers, Environmentally-induced displacements: the state of the art. Technical report, Environmentally-Induced Population Displacements and Environmental Impacts Resulting from Mass Migration, International Symposium (1996).

[29] G.I.P. Ottaviano, Monopolistic competition, trade, and endogenous spatial fluctuations. Regl. Sci. Urban Econ. 31 (2001) $51-77$.

[30] G.I.P. Ottaviano and G. Peri, Rethinking the gains from immigration: theory and evidence from the US. CEPR Discussion Papers 5226 (2005).

[31] T. Puu, A simplified model of spatiotemporal population dynamics. Environ. Plan. A 17 (1985) 1263-1269.

[32] T. Puu, On growth and dispersal of populations. Ann. Regl. Sci. 23 (1989) 171-186.

[33] S.C. Salop, Monopolistic competition with outside goods. Bell J. Econ. 10 (1979) 141-156.

[34] J.G. Skellam, Random dispersal in theoretical populations. Biometrica 38 (1951) 196-218.

[35] M. Smith, J. Sanchirico and J. Wilen, The economics of spatial-dynamic processes: applications to renewable resources. J. Environ. Econ. Manag. 57 (2009) 104-121.

[36] A. Turing, The chemical basis of morphogenesis. Philos. Trans. Roy. Soc. Lond. 237 (1952) 37-72.

[37] UNHCR, Global Report, available at http://www.unhcr.org/gr11/index.xml (2011).

[38] J.H. von Thünen, Von Thunen's isolated state: an English edition of Der isolierte Staat. Pergamon Press (1966).

[39] V. Volpert, S. Petrovskii and A. Zincenko, Interaction of human migration and wealth distribution. Nonlin. Anal. 159 (2017) 408-423. 